\title{
Correlative intravital imaging of cGMP signals and vasodilation in mice
}

\section{Martin Thunemann ${ }^{1}$, Kjestine Schmidt ${ }^{2}$, Cor de Wit ${ }^{2}$, Xiaoxing Han ${ }^{3}$, Rakesh K. Jain ${ }^{3}$, Dai Fukumura ${ }^{3}$ and Robert Feil ${ }^{1 *}$}

1 Interfakultäres Institut für Biochemie, University of Tübingen, Tübingen, Germany

2 Institut für Physiologie, Universität zu Lübeck, Lübeck, Germany

${ }^{3}$ Edwin L. Steele Laboratory, Department of Radiation Oncology, Massachusetts General Hospital and Harvard Medical School, Boston, MA, USA

Edited by:

Gil Bub, University of Oxford, UK

Reviewed by:

Irena Levitan, University of Illinois at

Chicago, USA

Erik Fung, University of Southern

California, USA

Andreas Koschinski, University of

Oxford, UK

*Correspondence:

Robert Feil, Interfakultäres Institut für Biochemie, University of

Tübingen, Hoppe-Seyler-Str. 4,

72076 Tübingen, Germany

e-mail: robert.fei@@uni-tuebingen.de
Cyclic guanosine monophosphate (cGMP) is an important signaling molecule and drug target in the cardiovascular system. It is well known that stimulation of the vascular nitric oxide (NO)-cGMP pathway results in vasodilation. However, the spatiotemporal dynamics of cGMP signals themselves and the cGMP concentrations within specific cardiovascular cell types in health, disease, and during pharmacotherapy with cGMP-elevating drugs are largely unknown. To facilitate the analysis of cGMP signaling in vivo, we have generated transgenic mice that express fluorescence resonance energy transfer (FRET)-based cGMP sensor proteins. Here, we describe two models of intravital FRET/cGMP imaging in the vasculature of cGMP sensor mice: (1) epifluorescence-based ratio imaging in resistance-type vessels of the cremaster muscle and (2) ratio imaging by multiphoton microscopy within the walls of subcutaneous blood vessels accessed through a dorsal skinfold chamber. Both methods allow simultaneous monitoring of NO-induced cGMP transients and vasodilation in living mice. Detailed protocols of all steps necessary to perform and evaluate intravital imaging experiments of the vasculature of anesthetized mice including surgery, imaging, and data evaluation are provided. An image segmentation approach is described to estimate FRET/cGMP changes within moving structures such as the vessel wall during vasodilation. The methods presented herein should be useful to visualize cGMP or other biochemical signals that are detectable with FRET-based biosensors, such as cyclic adenosine monophosphate or $\mathrm{Ca}^{2+}$, and to correlate them with respective vascular responses. With further refinement and combination of transgenic mouse models and intravital imaging technologies, we envision an exciting future, in which we are able to "watch" biochemistry, (patho-)physiology, and pharmacotherapy in the context of a living mammalian organism.

Keywords: biosensor, cremaster, cyclic GMP, dorsal skinfold chamber, fluorescence resonance energy transfer intravital imaging, microcirculation, multiphoton microscopy

\section{INTRODUCTION}

Blood vessels are highly organized structures consisting of various cell types including endothelial cells in the intima, smooth muscle cells (SMCs) in the media, and fibroblasts in the adventitia. These cells receive signals from surrounding tissue, blood, and the nervous system, and they interact with each other in multiple ways. Using ex vivo or in vitro systems, it is challenging, if not impossible, to mimic the vessel's three-dimensional structure, microenvironment, and physiological control systems present in vivo. Current research aims to understand biochemical pathways contributing to vascular function and dysfunction in health and disease. Intravital microscopy (IVM) is a powerful tool to study signaling processes in vascular cells while still in their natural environment. IVM-based approaches have been developed to analyze vascular structure and hemodynamic parameters in vivo, mainly in surgically exposed tissues or through implanted optical windows (Weigert et al., 2013). Technical innovations in confocal and multiphoton (MP) microscopy and the generation of transgenic animals expressing fluorescent proteins paved the way for state-of-the-art IVM (Jain et al., 2002; Kirkpatrick et al., 2012). In combination with motion compensation, IVM can achieve spatiotemporal resolutions similar to microscopy under in vitro conditions (Lee et al., 2014).

$\mathrm{Ca}^{2+}$, cyclic adenosine monophosphate (cAMP), and cyclic guanosine monophosphate (cGMP) are key players in the regulatory network of vascular function (Carvajal et al., 2000; De Wit et al., 2000; Somlyo and Somlyo, 2000; Berridge, 2008; Morgado et al., 2012). Transgenic mice were generated that express fluorescent $\mathrm{Ca}^{2+}$ indicator proteins in endothelial cells or SMCs (Isotani et al., 2004; Tallini et al., 2007; Mauban et al., 2013) and cardiomyocytes (reviewed in Kaestner et al., 2014). Interestingly, Mauban et al. recently reported that spontaneous high-amplitude $\mathrm{Ca}^{2+}$ waves do not occur in SMCs of resistancetype vessels in vivo, although these waves have been observed 
before in isolated vessels of various origin (Mauban et al., 2013). This finding challenges the hypothesis that high-amplitude $\mathrm{Ca}^{2+}$ waves significantly contribute to the regulation of basal vascular tone in vivo (Mauban et al., 2013) and highlights the importance to study cardiovascular physiology in vivo (Wier, 2014). In contrast to $\mathrm{Ca}^{2+}$, vascular cAMP and cGMP signals have not been visualized in vivo until now, although respective biosensors have been developed and used in cells and isolated tissues (Zaccolo et al., 2005; Nikolaev and Lohse, 2009; Thunemann et al., 2013a). These experiments indicated the existence of subcellular cyclic nucleotide compartments in cardiomyocytes and vascular SMCs and a cross-modulation of cAMP and cGMP signals in vitro (Fischmeister et al., 2006; Zaccolo and Movsesian, 2007; Nausch et al., 2008; Stangherlin and Zaccolo, 2012). Localized signaling domains might play important roles in cardiovascular function and dysfunction (Nikolaev et al., 2010). In vitro experiments have also shown substantial crosstalk between $\mathrm{Ca}^{2+}$ and cyclic nucleotide signaling pathways (Carvajal et al., 2000; Feil et al., 2003). However, the relevance of these mechanisms for the regulation of vascular tone in vivo has yet to be demonstrated.

Cyclic GMP is generated by guanylyl cyclases in response to nitric oxide (NO) and natriuretic peptides. Its effects are mediated through cGMP-dependent protein kinases (cGKs), cyclic nucleotide-gated ion channels, and cyclic nucleotide-degrading phosphodiesterases. A key function of cGMP in the vasculature is the induction of vasodilation, perhaps by modulating $\mathrm{Ca}^{2+}$ signaling pathways in vascular SMCs (Lincoln et al., 2001; Beavo and Brunton, 2002; Hofmann et al., 2006; Kemp-Harper and Feil, 2008; Francis et al., 2011). Indeed, IVM of the cremaster microcirculation demonstrated the importance of the NO-cGMP-cGK pathway for dilation of resistance-type vessels in vivo (De Wit et al., 1994; Koeppen et al., 2004). Moreover, natriuretic peptideinduced cGMP modulates endothelial permeability, therefore contributing to the regulation of blood volume and pressure (Kuhn, 2012). Cyclic GMP also mediates the effects of drugs used to treat angina pectoris (e.g., organic nitrates), erectile dysfunction (e.g., sildenafil, tadalafil), and pulmonary hypertension (e.g., sildenafil, riociguat) (Kemp-Harper and Feil, 2008; Schlossmann and Schinner, 2012). Although considerable progress has been made in unraveling cGMP's roles in cardiovascular physiology and pathophysiology, many of its functions remain controversial (Feil et al., 2003, 2005; Tsai and Kass, 2009).

To extend our understanding of cardiovascular cGMP signaling in vivo, we sought to establish methods that allow the visualization of cGMP in living mice. Then, we would be able to correlate cGMP signals with functional responses, such as vasodilation, under physiological and pathophysiological conditions, or upon pharmacotherapy with cGMP-elevating drugs. Therefore, we generated transgenic mice expressing cGMP biosensors based on fluorescence (or Förster) resonance energy transfer (FRET) and reported for the first time on intravital FRET/cGMP imaging in living mice (Thunemann et al., 2013b).

\section{METHODOLOGY}

This section describes the steps necessary to perform and evaluate IVM experiments in the vasculature of anesthetized mice expressing FRET-based cGMP biosensors. We also describe an evaluation routine that allows estimating FRET/cGMP changes in moving structures, such as dilating vessels. These methods are likely adaptable to experiments within other vascular beds, e.g., in mesenteric, femoral, or cerebral arteries, or with mice expressing other FRET-based biosensors.

\section{CGMP BIOSENSOR}

We use the FRET-based cGMP biosensor cGi500 ("cGMP indicator with an $\mathrm{EC}_{50}$ of $500 \mathrm{nM}$ ") generated by Russwurm et al. (2007). This monomolecular biosensor consists of the two cGMPbinding sites from bovine cGK type I sandwiched between cyan and yellow fluorescent protein (CFP and YFP). Detection of cGMP with cGi500 is based on FRET from CFP to YFP, whose spectra show sufficient overlap for efficient FRET (Figure 1A). In the absence of cGMP, CFP and YFP are in favorable distance and orientation for FRET to occur, whereas cGMP binding induces a reversible structural change of cGi500 that reduces FRET efficiency (Figure 1B). The ratio of CFP and YFP fluorescence $(\mathrm{R}=\mathrm{CFP} / \mathrm{YFP})$ recorded upon CFP excitation can be taken as a measure for the FRET efficiency (Clegg, 2009) and, therefore, for intracellular cGMP concentrations measured with cGi500. It has been shown in sensor-containing cell extracts and escinpermeabilized cells that cGi500 detects cGMP concentrations as low as $50-100 \mathrm{nM}$ and is saturated at approx. $3 \mu \mathrm{M}$ cGMP. Similar experiments have shown that cGi500 detects cAMP only at levels higher than $30 \mu \mathrm{M}$, which exceeds typical physiological cAMP concentrations (Russwurm et al., 2007; Thunemann et al., 2013a; Milde and Feil, unpublished results). Based on its high selectivity for cGMP over cAMP, its dynamic range for cGMP detection, fast kinetics, and relatively large CFP/YFP ratio changes of up to $\sim 50 \%$, the cGi500 sensor appears to be suitable for in vivo use.

\section{TRANSGENIC MICE}

Animal experiments were performed in compliance with the guidelines for human care and use of laboratory animals. They were approved by the Regierungspräsidium Tübingen, the Ministerium für Energiewende, Landwirtschaft, Umwelt und ländliche Räume des Landes Schleswig-Holstein, and local authorities in Boston, MA. The generation of transgenic cGMP sensor mice has been described previously (Thunemann et al., 2013b). Transgene structures are shown in Figure 1C. Briefly, the SM22-cGi500 mouse line was generated by random transgenesis using a 445-bp promoter fragment of the Transgelin/SM22 gene (Li et al., 1996) to drive cGi500 expression. The R26-CAGcGi500(L2) and R26-CAG-cGi500(L1) lines were generated by targeted mutagenesis of the Rosa26 (R26) locus with a construct derived from the R26-mT/mG targeting vector (Muzumdar et al., 2007). IVM data shown and discussed in this article were obtained with the R26-CAG-cGi500(L1) mouse line that expresses cGi500 ubiquitously (Thunemann et al., 2013b).

\section{EPIFLUORESCENCE cGMP IMAGING IN THE CREMASTER MICROCIRCULATION \\ Imaging setup}

- Microscope: upright Axioplan II FS (Carl Zeiss Microscopy) with Achroplan $40 \times / 0.75$ N.A. water immersion lens. The 


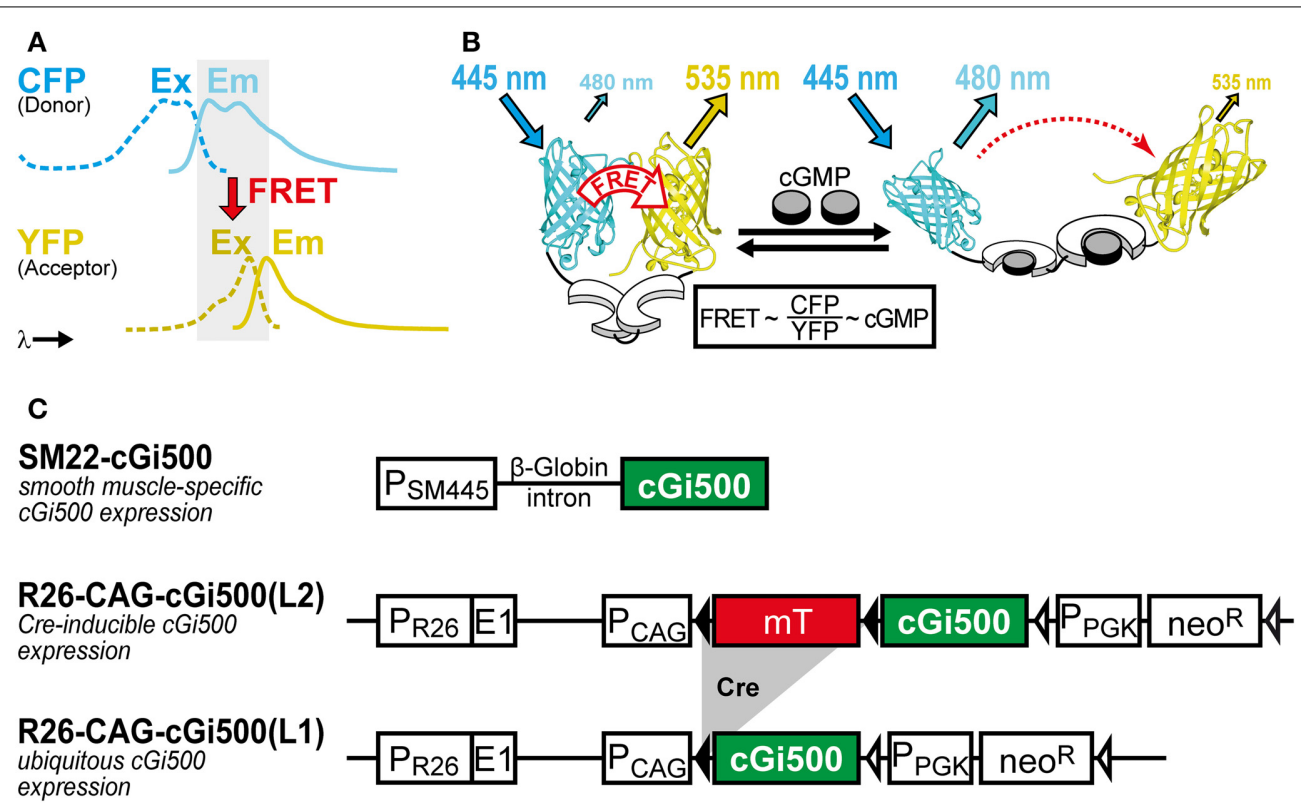

FIGURE 1 | (A,B) Working principle of the FRET-based cGi500 biosensor and (C) transgenes used to generate cGi500-expressing mice. (A) Spectral overlap (gray) of YFP excitation (Ex, dashed lines) and CFP emission (Em, solid lines) spectra that is necessary for FRET to occur. (B) The cGMP indicator protein cGi500 consists of the tandem cGMP-binding sites from bovine cGK type I (white) flanked by CFP and YFP. Without CGMP, FRET occurs from excited CFP to YFP, leading to light emission from YFP. Binding of CGMP (gray) causes a conformational change and a decrease in FRET efficiency, so that light emission from YFP at $535 \mathrm{~nm}$ is reduced and emission from CFP at $480 \mathrm{~nm}$ is increased. (B) is reproduced from
Thunemann et al. (2013b). (C) Constructs used to generate transgenic cGi500-expressing mice. Abbreviations: E1, first exon of the endogenous Rosa26 gene; $\mathrm{mT}$, membrane-targeted tandem-dimer tomato red fluorescent protein; neo ${ }^{R}$, neomycin resistance gene; $P_{C A G}$, chicken actin/ $\beta$-globin promoter; $P_{\text {PGK }}$, phosphoglycerate kinase promoter; $P_{\mathrm{SM} 445}$, 445-bp promoter fragment of the Transgelin/SM22 gene; $P_{R 26}$,

endogenous Rosa26 promoter. Black triangles represent loxP sites, open triangles represent FRT sites. See also Section "Selection of Appropriate Biosensors and Generation of Biosensor-Expressing Mice" for further details on mouse generation and characterization. system is placed on a motorized shifting table (Luigs and Neumann).

- Light source: Polychrome V (FEI Munich) set to $420 \mathrm{~nm}$ or Spectra X with 438/24 nm light engine (Lumencor).

- Filters: $470 \mathrm{~nm}$ dichroic mirror (AHF). Beam splitter: MicroImager DUAL-View with $516 \mathrm{~nm}$ dichroic mirror, 480/50 and 535/40 nm emission filters (Photometrics).

- Detector: Andor iXon 885 (Andor Technology) or Quant-EM 512sc (Photometrics) EM-CCD camera.

- Software: Live Acquisition (FEI Munich) or VisiView (Visitron Systems).

\section{Surgery}

All surgical procedures have to be approved by the Institutional Animal Care and Use Committee. Male mice are required for IVM of the cremaster microcirculation.

(1) Anesthesia. Add $0.2 \mathrm{~mL}$ midazolam $(5 \mathrm{mg} / \mathrm{mL}$, MidazolamHameln, Hameln Pharmaceuticals), $0.1 \mathrm{~mL}$ dexmedetomidin $(0.5 \mathrm{mg} / \mathrm{mL}$ Dexdomitor, Lilly Germany), and $0.2 \mathrm{~mL}$ fentanyl $(0.05 \mathrm{mg} / \mathrm{mL}$ Fentanyl-Janssen, Janssen-Cilag) to $2.15 \mathrm{~mL}$ Ringer's salt solution (final concentrations: $378 \mu \mathrm{g} / \mathrm{mL}$ midazolam, $19 \mu \mathrm{g} / \mathrm{mL}$ dexmedetomidin, $3.8 \mu \mathrm{g} / \mathrm{mL}$ fentanyl). The animal is anesthetized by intraperitoneal injection of this solution $(13 \mathrm{~mL} / \mathrm{kg}$ body weight) followed by continuous infusion via a jugular vein catheter at a rate of $3 \mathrm{~mL} / \mathrm{h} / \mathrm{kg}$ body weight with a syringe pump (78-9100 W, World Precision Instruments). The infusion rate should be adjusted depending on the sedative effect monitored by provocation tests. If the animal reacts to these tests, increase the infusion rate initially to $4 \mathrm{~mL} / \mathrm{h} / \mathrm{kg}$ body weight, followed by a rate of $3.3 \mathrm{~mL} / \mathrm{h} / \mathrm{kg}$ body weight until the animal no longer reacts.

(2) Preparation of surgery. After injection of anesthetics, wait $20 \mathrm{~min}$ before shaving the right scrotum, the inguinal region, and the ventral neck region using an electric clipper (GT 420 ISIS, Aeskulap). If the animal still reacts to this manipulation, apply another intraperitoneal injection of anesthetics ( $5 \mathrm{~mL} / \mathrm{kg}$ body weight). Place the animal in supine position on a Styrofoam stage that allows temporary fixing of tubes and threads by needles. Maintain and monitor the animal's body temperature by using an electric heating mat and a temperature monitoring system (Telemeter Electronic).

(3) Endotracheal tube for mechanical ventilation. Open the neck by a ventral midline incision of $\sim 25 \mathrm{~mm}$ length using scissors. The salivary glands covering the trachea are gently pushed aside and the trachea is isolated by blunt preparation and enlaced with two threads. Use these threads to pull the trachea slightly upwards and cut it halfway through with a scalpel to allow the insertion of a tube $(15 \mathrm{~mm}$ length, I.D. $0.86 \mathrm{~mm}$, O.D. $1.27 \mathrm{~mm}$, polyethylene, Portex, Smiths 
Medical International). Push the tube $5 \mathrm{~mm}$ down the trachea and fix it using the two threads. Ensure that the animal is still breathing and that the knots secure but do not occlude the tube. After the animal has been moved to the imaging setup, connect the tube to the ventilator (Minivent Type 845, Hugo Sachs Elektronik) and start mechanical ventilation (see Section "Imaging”).

(4) Jugular vein catheter for intravenous infusion of anesthetics. Locate the jugular vein right to the trachea, clean it carefully from surrounding tissue by blunt preparation, and enlace it using three threads (Figure 2A, inset). Place one thread caudally toward the heart and put it under little tension to obstruct blood flow. The second thread is then positioned cranially, closed, and knotted to obtain a sufficient length of vein between these threads to introduce the catheter tube. Put little tension onto both threads by fixing them temporarily with needles placed into the Styrofoam. Use fine scissors (Fine Science Tools) to open the jugular vein halfway. Use a 27 -gauge cannula $(0.40 \times 20 \mathrm{~mm}$, Sterican, Braun $)$ bend by $90^{\circ}$ and attached to a $1-\mathrm{mL}$ syringe to open the vein. Carefully insert a saline-filled polyethylene tube (I.D. $0.26 \mathrm{~mm}$, O.D. $0.61 \mathrm{~mm}$, Portex, Smiths Medical International) beneath the cannula. Release the caudal thread, push the tube about $10 \mathrm{~mm}$ toward the heart, and secure the catheter using all three threads. Usually it is possible to verify correct positioning by blood aspiration. Close the wound by three single cutaneous sutures and start continuous infusion of anesthetics at a rate of $3 \mathrm{~mL} / \mathrm{h} / \mathrm{kg}$ body weight as described above.

(5) Cremaster preparation. Place the mouse in supine position on a custom-made animal stage that is later used for IVM (Figure 2A). Push the right testicle down into the scrotum. Cut a hole into the scrotum at its peak using scissors, insert one blade, and open the scrotum along its length toward the inguinal region. The cremaster muscle is now visible and requires continuous moistening; start dropping warmed $\left(34^{\circ} \mathrm{C}\right)$ salt solution at a rate of $3 \mathrm{~mL} / \mathrm{min}$ from a reservoir onto the muscle. The salt solution contains (in $\mathrm{mmol} / \mathrm{L}$ ): $118.4 \mathrm{NaCl}, 20 \mathrm{NaHCO}_{3}, 3.8 \mathrm{KCl}, 2.5 \mathrm{CaCl}_{2}, 1.2 \mathrm{KH}_{2} \mathrm{PO}_{4}$, and $1.2 \mathrm{MgSO}_{4}$; it is gassed with $5 \% \mathrm{CO}_{2}$ and $95 \% \mathrm{~N}_{2}$ to adjust the $\mathrm{pH}$ to 7.4. Mobilize the peak of the muscle pouch and secure it using an atraumatic thread (Serapren $6 / 0$, Serag-Wiessner). Establish a constant fluid flow over the muscle (superfusion) for $10 \mathrm{~min}$ to soak and visualize connective tissue. Then, carefully clean the muscle from this connective tissue. Do not cut into muscle tissue and use the initially placed thread to elevate the cremaster to access its bottom side. Now, the muscle pouch is spread over the glass window that is part of the stage. Around this window, a silicon wall holds the threads fixing the edges of the muscle (Figure 2A). Pull the thread attached to the cremaster's peak through the silicon wall and apply some tension to pull the muscle pouch over the window. Use scissors to cut a small hole into the pouch at its peak, insert one blade, and cut it open up to the inguinal region. Avoid cutting through larger vessels that usually lay at the bottom of the pouch. Use 2-3 atraumatic threads at each side to fix the lateral edges of the muscle; apply some tension to flatten the cremaster over the glass window (Figure 2A). Cut the ligament between cremaster and testis to allow repositioning of the testis into the abdominal cavity, which requires cutting one pair of a larger arteriole and vein that enters through this ligament into the testis. Subsequent bleeding will usually cease after a short period. Hemostasis can also be achieved using collagen-based hemostatic sponges.

(6) Isolation of arterioles. For particular experiments, arterioles can be freed from surrounding striated muscle (see Result Section "Imaging of cGMP within the Cremaster Microcirculation" and Figure 2C). This procedure requires a surgical microscope with 40-fold magnification (Wild Heerbrugg) and fine preparation tools. Cut into the skeletal fibers that lay on top of the vessels and strip the fibers away using fine forceps. Only isolate small (1-2 mm) arteriole sections for better viability.

\section{Imaging}

(1) Place the animal stage onto the microscope and move the cremaster muscle into the light path. Do not interrupt superfusion of salt solution for longer than $30 \mathrm{~s}$. Attach the ventilator and start mechanical breathing with a frequency of 160 per min and a stroke volume of $225 \mu \mathrm{L}$. Check connections of the jugular vein catheter and the level of anesthesia.

(2) Move the objective lens to working distance and adjust the rate of superfusion to $8 \mathrm{~mL} / \mathrm{min}$. The solution is delivered onto the water immersion objective to form a flowing "drop" between lens and specimen. To provide a physiologic environment, the superfusate is kept at low oxygen tension by gassing with $5 \% \mathrm{CO}_{2} / 95 \% \mathrm{~N}_{2}$ before and throughout the experiment as well as by using gas-impermeable plastic or glass tubes. Gravity-driven superfusion from a reservoir avoids pulsatile flow, but care has to be taken that the rate of superfusion stays constant throughout the experiment, particularly if drugs are applied through a roller pump (Miniplus3, Gilson). The roller pump delivers drugs into the main superfusion tube just before the fluid reaches the objective. The delivery rate of the roller pump is set to $0.08 \mathrm{~mL} / \mathrm{min}$. Thus, drugs delivered by this pump are diluted 100 -fold just before they reach the specimen. Vehicle (e.g., water) should be delivered if no drug is applied. Check for continuous removal of the superfusate to avoid leakage into the microscope.

(3) Select an appropriate region using bright field and fluorescence illumination. Identify vessel types by diameter, wall structure, and direction of blood flow that diverges in branching arterioles but converges in branching venules.

(4) Select illumination time and intensity, as well as camera gain and binning to obtain images with sufficient signalto-noise ratios, while avoiding excessive photobleaching. Settings depend on the imaging setup as well as the biosensor that is used, and its expression level. Parameters have to be optimized for individual setups and according to experimental demands (e.g., temporal vs. spatial resolution). Representative acquisition parameters for the setup described in Section "Imaging setup" are given in the legend to Figure 2F. 

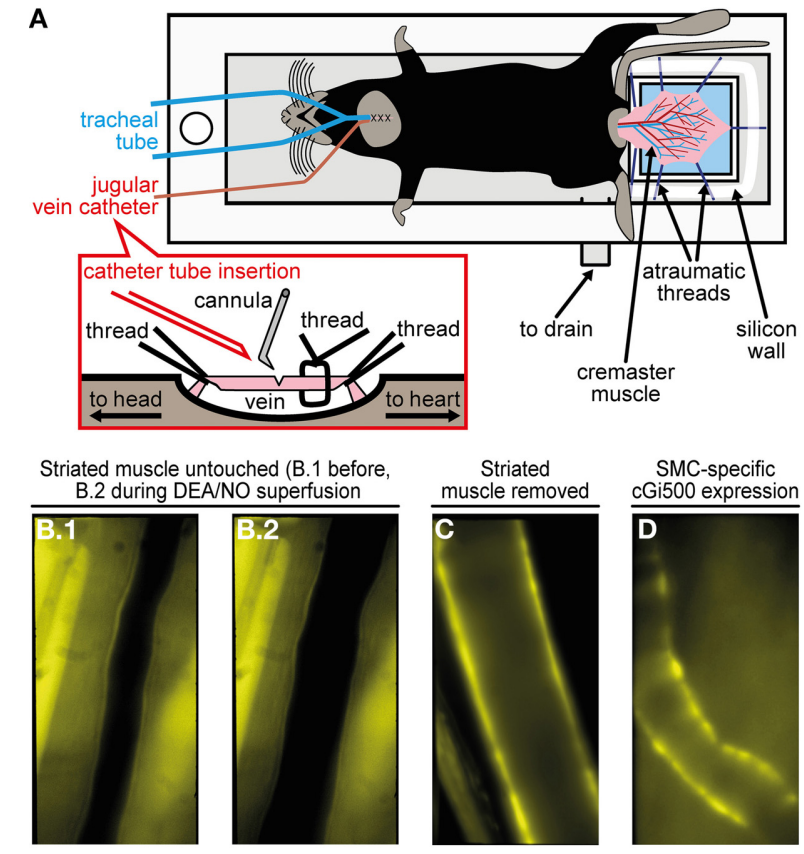

SMC-specific

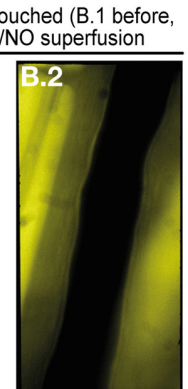

cGMP imaging (striated muscle removed)
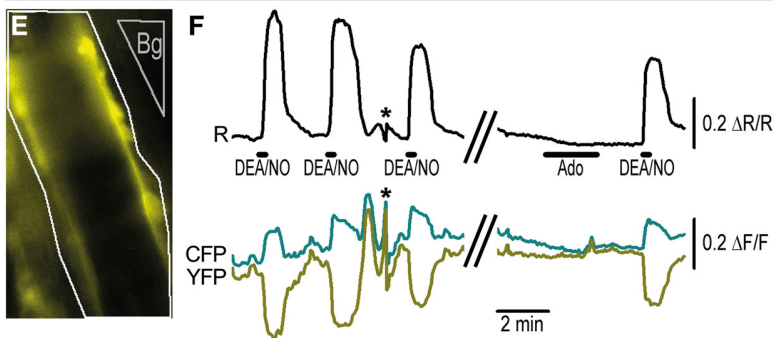

FIGURE 2 | Imaging of cGMP within the cremaster microcirculation. (A) A custom-made acrylic glass stage holds the anesthetized mouse in supine position on a heating pad (not shown). The cremaster muscle has been surgically exteriorized and fixed with atraumatic threads on top of a coverslip (light blue). Threads are pinned through a silicone wall that surrounds the coverslip as a semicircle. The cremaster is superfused with pre-warmed $\left(34^{\circ} \mathrm{C}\right)$ salt solution delivered along the objective lens (not shown) approaching the cremaster from top. The solution is drained through the outlet at the bottom of the stage. For mechanical ventilation, a tube has been inserted into the trachea. A detailed depiction of jugular vein cannulation is shown in the inset. (B) Arteriole within the intact cremaster muscle of a R26-CAG-cGi500(L1) mouse. B.1 shows the vessel before and B.2 during superfusion of $10 \mu \mathrm{M}$ DEA/NO that causes vasodilation.

Estimation of cGMP levels was not possible under this condition, because strong fluorescence from striated muscle prevented FRET measurements within the vessel wall. (C) After removal of striated muscle, walls of cremaster arterioles of a R26-CAG-cGi500(L1) mouse are clearly visible, but they lost their basal tone and did not dilate upon superfusion with DEA/NO or other vasoactive drugs. (D) In an intact cremaster preparation of a R26-CAG-cGi500(L2); SMA-CreER ${ }^{\text {T2 }}$ double-transgenic mouse, only the vessel walls show cGi500 expression. The animal was treated for 5 days with $1 \mathrm{mg}$ tamoxifen (i.p.) per day to induce $\mathrm{CreER}^{\mathrm{T} 2}$-mediated activation of the R26-CAG-cGi500(L2) transgene specifically within SMCs. The image was taken 1 week after the last tamoxifen injection. (E) Definition of ROls (arteriole: white outline; background region: gray, "Bg") for cGMP imaging in the cremaster of a R26-CAG-cGi500(L1) mouse after removal of striated muscle. (F) Cyclic GMP imaging of the arteriole shown in (E); the black trace indicates baseline-normalized CFP/YFP ratio changes $(\Delta R / R)$

(Continued)

\section{FIGURE 2 | Continued}

representing intracellular cGMP levels; cyan and yellow traces represent baseline-normalized CFP and YFP fluorescence intensity changes $(\Delta F / F)$. Repeated application of $10 \mu \mathrm{M}$ DEA/NO induced reversible cGMP transients, while $10 \mu \mathrm{M}$ adenosine (Ado), known to induce relaxation via the cAMP pathway, did not induce detectable ratio changes. Antiparallel changes of CFP and YFP intensities are indicative for true changes of cGi500 FRET efficiency. Asterisks denote artifacts due to focus drift. Acquisition parameters were as follows: excitation: Polychrome $V$ at $420 \mathrm{~nm}$; objective: $40 \times$; acquisition frequency: one image every $0.5 \mathrm{~s}$; camera: Andor iXon 885 with $2 \times 2$ binning $(251 \times 501$ pixels $)$ and gain set to 25; exposure time: $150 \mathrm{~ms}$. Temporal binning of six frames was done before evaluation performed by subtraction of a background region. Evaluation via image segmentation led to similar results but lower $\Delta R / R$ values (not shown). All images (B-E) were obtained at $40 \times$ original magnification. Panels $\mathrm{E}$ and $\mathrm{F}$ are reproduced from Thunemann et al. (2013b) and show representative results from experiments with three animals.

(5) Acquire time series under basal conditions to estimate signalto-noise levels and to observe potential baseline drift or basal activity of the cells under investigation.

(6) After a stable baseline is recorded for a sufficient period (e.g., for 30-60 frames), drugs (e.g., the NO-releasing drug $\mathrm{DEA} / \mathrm{NO}$ ) can be applied via superfusion using the roller pump. Exchange vehicle with drug solution to start application and back to vehicle to terminate application. The time delay until the drug reaches the specimen can be estimated by sucking air into the tube of the roller pump. Observe the air bubble moving through the tube and measure the time until its delivery.

(7) During the imaging session, check anesthesia and vital parameters from time to time. Based on our experience, IVM can be performed for up to $6 \mathrm{~h}$. At the end of the experiment, apply a lethal dose of pentobarbital $(1 \mathrm{~g} / \mathrm{kg}$ body weight, e.g., $6.25 \mathrm{~mL} / \mathrm{kg}$ body weight of a $160 \mathrm{mg} / \mathrm{mL}$ pentobarbital solution, Narcoren, Merial) through the jugular vein catheter.

\section{MULTIPHOTON cGMP IMAGING IN SUBCUTANEOUS VESSELS USING THE DORSAL SKINFOLD CHAMBER Imaging setup}

Details on this IVM setup have been described previously (Brown et al., 2001).

- Microscope: upright BX51WI (Olympus) with Fluoview FV300 scanning system and $20 \times / 0.95$ N.A. water immersion lens. The system is placed on a vibration-free table (Melles Griot) within a light-tight enclosure.

- Light source: MaiTai Ti:Sapphire laser (Spectra-Physics) providing $100 \mathrm{fs}$ pulses at $80 \mathrm{MHz}$ repetition rate and $850 \mathrm{~nm}$ wavelength with a power of $36 \mathrm{~mW}$ at the sample surface.

- Filters: $750 \mathrm{~nm}$ short pass dichroic mirror (Chroma). FRET filter set: $505 \mathrm{~nm}$ long pass dichroic mirror (Chroma), 483/32 nm and 542/27 nm emission filters (Semrock).

- Detection: HC125-02 photomultiplier tubes (Hamamatsu Photonics).

- Software: FluoView (Olympus). 


\section{Surgery}

All surgical procedures have to be approved by the Institutional Animal Care and Use Committee. The procedure follows the protocol for implantation of dorsal skinfold chambers (DSCs) in mice as described in Leunig et al. (1992).

(1) Anesthetize mice by intraperitoneal injection of $90 \mathrm{mg} / \mathrm{kg}$ body weight ketamine and $9 \mathrm{mg} / \mathrm{kg}$ body weight xylazine. Wait $\sim 15$ min until the animal does not react to provocation tests anymore. During the surgical procedure, maintain the animal's core body temperature at $36-37^{\circ} \mathrm{C}$ using a heating pad. Apply a lubricant to prevent corneal dehydration.

(2) Shave and depilate the entire back of the animal prior to chamber implantation.

(3) Gently stretch the double layer of the skin from the back of the mouse and implant two symmetrical titanium frames (weight $3.2 \mathrm{~g}$; produced at the workshop of the Department of Radiation Oncology, MGH) making mirror images of each other to sandwich the skin. Place sutures in the top part of the skin through the holes at the top edge of the titanium frames to hold the frames in place.

(4) Remove one layer of the skin in a circular area of $15 \mathrm{~mm}$ in diameter. To stop bleeding, apply gentle pressure using a cotton swab. Cover the remaining layer consisting of epidermis, subcutaneous tissue, and striated muscle with a glass coverslip incorporated into a depression in one of the frames. Hold the coverslip in place with a retaining ring (Figure 3A).

(5) Allow the animal to recover for at least $48 \mathrm{~h}$. For analgesia, administer $0.05-0.1 \mathrm{mg} / \mathrm{kg}$ body weight buprenorphine every $12 \mathrm{~h}$ subcutaneously for 3 days, and thereafter as needed. Animals loose about $15 \%$ of their body weight within the first $48 \mathrm{~h}$ after DSC implantation and further weight loss or other signs of distress are usually not observed (Leunig et al., 1992). If there is any sign of inflammation, redness, fluid accumulation, edema, etc. in the DSC, animals are further observed for recovery or excluded from the study.

\section{Imaging}

IVM has to be performed with approval by the Institutional Animal Care and Use Committee.

(1) Anesthetize a mouse with DSC by subcutaneous or intraperitoneal injection of ketamine/xylazine $(90 / 9 \mathrm{mg} / \mathrm{kg}$ body weight). Alternatively, gas anesthesia such as $1-1.5 \%$ isoflurane in medical air can be used. Properly maintained gas anesthesia is more stable and suitable for long and/or physiological imaging studies.

(2) Place the anesthetized animal on a heated microscope stage. Apply a lubricant to prevent corneal dehydration. Clamp the chamber to a custom-built metal frame on the microscope stage. For drug application, place an intravenous catheter connected to a $1-\mathrm{mL}$ syringe filled with $0.9 \% \mathrm{NaCl}$ into the tail vein. The catheter consists of a 30-gauge needle connected to polyethylene tubing (PE-10, BD Medical, I.D. $0.28 \mathrm{~mm}$, O.D. $0.61 \mathrm{~mm}$ ) of appropriate length (see below). Fix the catheter needle with a small drop of superglue to the tail and the tubing with adhesive tape to the microscope stage.
(3) Place the animal-bearing stage on the imaging setup. The syringe connected to the catheter must later be accessible without disturbing image acquisition. Place a drop of water on the coverslip of the DSC and move the objective into working distance. Select an appropriate region under bright field illumination. Check the animal's vital parameters and close the enclosure. Examine the field of view under fluorescence illumination and fine-adjust $\mathrm{x} / \mathrm{y}$-position and the $\mathrm{z}$ focus.

(4) Prepare image acquisition: adjust illumination intensity, scan speed, zoom, and photomultiplier voltage to obtain images with sufficient signal-to-noise ratios. Image acquisition may not cause excessive bleaching of the biosensor. Settings have to be optimized according to the IVM setup, the respective biosensor and its expression level, as well as experimental demands (e.g., temporal vs. spatial resolution). Representative acquisition parameters for the setup described in Section "Imaging setup" are given in the legend to Figure 3E.

(5) Acquire time series under basal conditions to estimate signalto-noise levels and to observe potential baseline drifts or basal activity of the cells under investigation.

(6) After a stable baseline is recorded for a sufficient period (e.g., for 20-30 frames), substances are applied through the tail vein catheter. Apply vehicle (saline or PBS) and then test compounds (e.g., the NO-releasing drug DEA/NO); consider the catheter's death volume.

(7) Ketamine/xylazine anesthesia typically lasts for 30-40 min; check regularly for vital functions and state of consciousness during the imaging session. If necessary, inject ketamine again; use half of the initial dose. The animal should not be anesthetized for more than $1 \mathrm{~h}$. After imaging, carefully remove the catheter from the tail and allow the animal to recover in its home cage. We recommend performing imaging experiments not more frequently than every other day.

\section{IMAGE SEGMENTATION WITH A BINARY MASK AND DATA EVALUATION}

Image processing is performed using the open source software ImageJ or ImageJ-derived software (Schneider et al., 2012). In establishing this protocol, the data were evaluated manually, although ImageJ and other software packages (e.g., Metamorph, IDL, Matlab) have built-in functions and routines for automation. Handling of time-lapse image series may require large amounts of free working memory; therefore, data evaluation should be performed on computers equipped with $\geq 8$ GB RAM.

(1) Image preprocessing. Save CFP and YFP time series as files readable by ImageJ (http://imagej.nih.gov/ij/) or ImageJderived distributions like Fiji (http://fiji.sc/Fiji). Open the time series and check for correct alignment of CFP and YFP channels. If necessary, apply corrections like manual or automatic alignment procedures (see, e.g., Kardash et al., 2011; Pitkeathly et al., 2012). Signal-to-noise ratios can be improved by spatial or temporal binning (method: "sum" or "average") that on the other hand will lead to loss of spatial or temporal resolution. Thereafter, intensities 

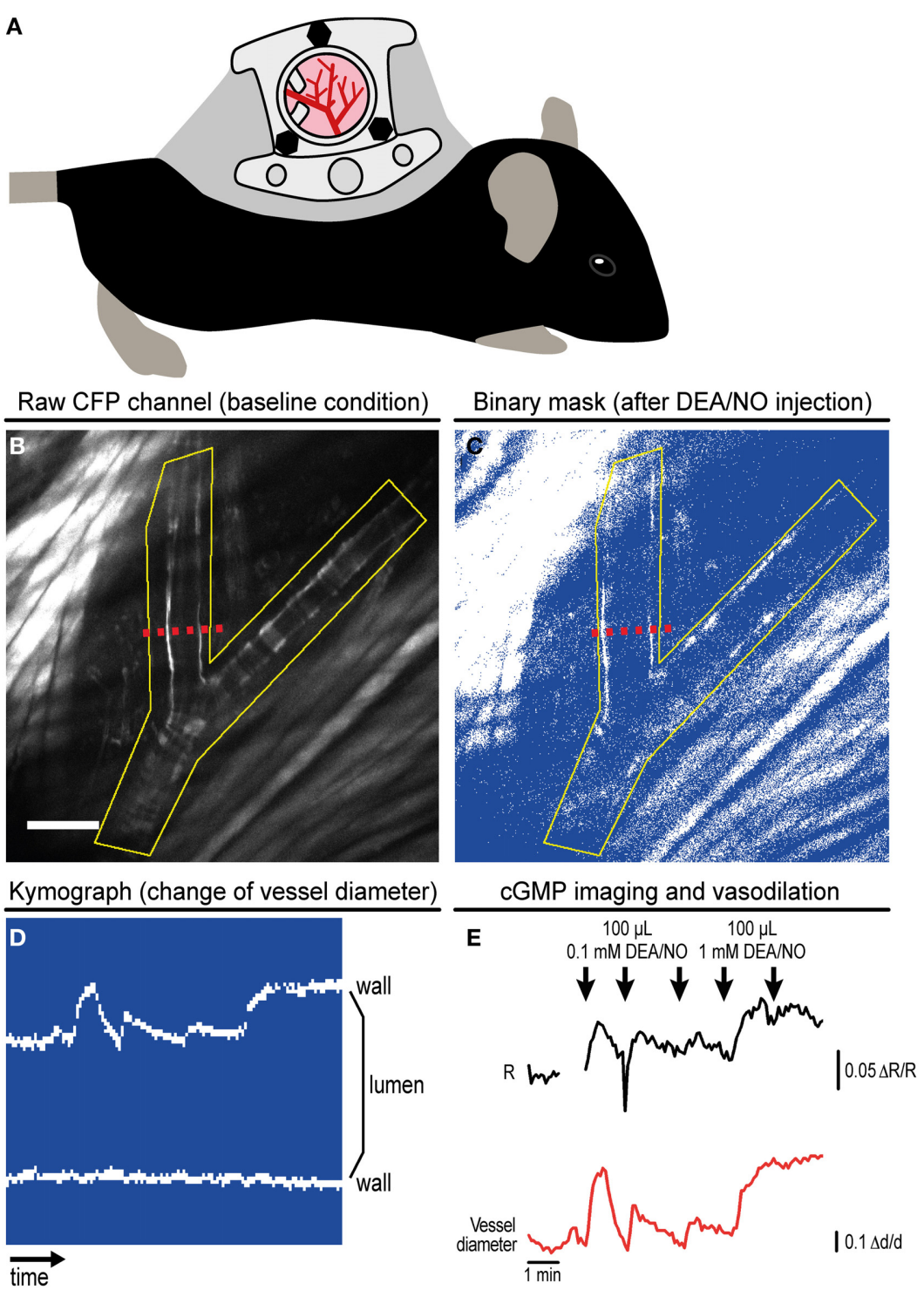

FIGURE 3 | Imaging of cGMP in subcutaneous vessels using the dorsal skinfold chamber (DSC). (A) Schematic drawing of a mouse with implanted DSC. (B) Field of view observed with MP microscopy through the DSC used for CGMP imaging as shown in (C-E). (B) shows the tissue before DEA/NO injection. Scale bar: $100 \mu \mathrm{m}$. (C) Image from the dynamic binary mask showing the tissue shortly after DEA/NO injection. (D) Kymograph showing movements of the vessel wall at the cross-section indicated by dashed red lines in (B,C) (time increases from left to right). Vasodilation was solely caused by movement of the upper vessel wall (left wall in $\mathbf{B}, \mathbf{C}$ ). (E) Cyclic GMP imaging of the (yellow) ROI outlined in (B,C). The black trace shows

changes of the baseline-normalized CFP/YFP ratio $(\Delta R / R)$ upon three intravenous injections of $0.1 \mathrm{mM}$ DEA/NO followed by two injections of $1 \mathrm{mM}$ DEA/NO. Few time points were omitted due to disturbance of image acquisition by ambient light. The relative vessel diameter change $(\Delta d / d$, red trace) was determined from the kymograph shown in (D). Acquisition parameters were as follows: excitation: $850 \mathrm{~nm}$ with $36 \mathrm{~mW}$ at the tissue surface; objective: $20 x$; acquisition frequency: one image every 5 s; image size: $512 \times 512$ pixels $(707.11 \times 707.11 \mu \mathrm{m})$. (B,E) are reproduced from Thunemann et al. (2013b). Representative results from five experimental sessions with three animals are shown from a background region can be subtracted and baseline normalization can be performed as described previously (Thunemann et al., 2013a). To account for tissue motion during image acquisition, we suggest performing image segmentation.

(2) Image segmentation with a dynamic binary mask. This routine derived from a strategy originally described by Zhang et al. (2010) is performed to follow CFP/YFP ratio changes in moving structures, such as the vessel wall during vasodilation. Subsequent steps and Figure 4 describe how the dynamic binary mask is generated und used:

- Apply the "threshold" function to the raw CFP or the raw YFP time series; select the lower threshold so that the structure of interest is above the threshold throughout the whole time series (perform "stack histogram function"). If CFP and YFP time series differ in quality (e.g., in signal-to-background ratios), use the series of higher 
quality, but make sure that decreased fluorescence caused by FRET efficiency changes do not cause the structure of interest to fall below the threshold.

- The final binary mask is generated by dividing the thresholded time series by itself using the "Image Calculator" function with the "32-bit (float) result" option enabled. The resulting dynamic binary mask has only values of " 1 " (pixels above threshold) or "NaN" for "not a number" (pixels below threshold).

- Apply this mask to both time series using the "Multiply" operation within the "Image Calculator" function. In the resulting segmented CFP and YFP time series, bright structures above the threshold possess intensity values as in the original time series, while dim/non-fluorescent structures below the threshold have "NaN" values (Figure 4).

- Note that background fluorescence (set to "NaN") is not subtracted from pixels above the threshold (see also discussion in Section "Multiphoton cGMP Imaging of Subcutaneous Vessels Using the Dorsal Skinfold Chamber").

(3) ROI definition and extraction of CFP and YFP intensities. Draw regions defining the target structure during the whole experiment considering shape changes and movements. As "NaN" pixels do not contribute to the region's average intensities, ROIs may contain large proportions of background fluorescence. Add regions to the "ROI Manager" and perform the "Multi Measure" command twice to extract mean intensities from both channels. Transfer values to Excel (Microsoft) or Origin (OriginLab) for further evaluation as described (Thunemann et al., 2013a).

(4) Generation of time-lapse ratio images. Pixel-by-pixel $\mathrm{CFP} / Y F P$ ratio series representing cGMP levels are calculated by dividing thresholded time series by each other using the "Image Calculator" function.

(5) Estimation of vessel diameter. To estimate changes in vessel diameter, draw a line orthogonal to the direction of blood flow into the dynamic binary mask. The line has to cover both vessel walls throughout the experiment. Generate a kymograph using the "Reslice" command within the "Stacks" menu with the "Rotate by 90 degrees" option enabled. The resulting image shows the time series at the crosssection of the vessel wall with time increasing from left to right (Figure 3D) and can be used for manual or semiautomatic vessel diameter estimation. Kymographs derived from CFP/YFP ratio series show changes in cGMP levels and vessel diameter within the same image.

\section{RESULTS AND DISCUSSION \\ SELECTION OF APPROPRIATE BIOSENSORS AND GENERATION OF BIOSENSOR-EXPRESSING MICE}

Two designs of genetically encoded biosensors are commonly used. One class includes monomolecular FRET-based biosensors like cGi-type cGMP sensors that contain donor and acceptor fluorophore in a single polypeptide chain. Other biosensors, such as FlincG-type cGMP sensors (Nausch et al., 2008; Bhargava et al., 2013) or $\mathrm{Ca}^{2+}$ sensors of the GCaMP family (Tian et al., 2009;
Chen et al., 2013), contain circularly permutated green fluorescent protein (cpEGFP). Monomolecular FRET-based biosensors can be measured by simultaneous acquisition of donor and acceptor emission upon donor excitation. In camera-based systems, beam splitters should be used to direct donor and acceptor fluorescence to two separate cameras or onto the halves of a single camera chip. In scanning microscopes, emitted light is detected by two separate photomultiplier tubes. Emission of cpEGFP-based biosensors can be measured at a single wavelength. Potential advantages and disadvantages of FRET- and cpEGFPbased biosensors are discussed in more detail in Newman et al. (2011), Kaestner et al. (2014). Ideally, any change in biosensor fluorescence should originate from ligand binding, but not from other processes that affect fluorescence intensity recordings, such as motion of the target structure. In this regard, ratiometric FRET-based biosensors are advantageous over cpEGFPbased biosensors, because not a single fluorescence intensity (e.g., EGFP) but the ratio between two simultaneously recorded fluorescence intensities (e.g., CFP/YFP) is taken to estimate ligand binding to the sensor. Artificial intensity changes will not affect quantification as long as both donor and acceptor fluorescence are affected to the same extent. This should be the case, for instance, upon movement of the imaged tissue due to vasodilation, heartbeat, blood flow, or breathing.

Biosensor-expressing transgenic mice are important tools for IVM. Expression of the biosensor must be appropriate for its detection with sufficient signal-to-noise ratios. Initially, we used random transgenesis to generate cGi500-expressing mice, which is a relatively simple method in terms of transgene assembly and mouse generation. However, it often requires screening of many founder lines to identify lines with stable and sufficient transgene expression. Using an appropriate promoter is critical for successful generation of such a transgenic mouse line. Unfortunately, random integration of the transgene into the genome often leads to unpredictable and unwanted expression patterns. We used the 445-bp promoter fragment of the Transgelin/SM22 gene (Li et al., $1996)$ to generate SM22-cGi500 mice with SMC-specific cGi500 expression (Figure 1C upper). Among 11 founder lines, one mouse line showed stable SMC-specific cGi500 expression (data not shown). The expression level of cGi500 in SMCs derived from aorta, colon, and bladder is heterogeneous with $\sim 25 \%$ of the cells showing relatively strong cGi500 expression (Thunemann et al., 2013b). However, SM22-cGi500 mice do not express detectable cGi500 levels in cremaster arterioles (Thunemann et al., unpublished data), so that these mice cannot be used for cGMP imaging in the cremaster microcirculation.

To generate mice with strong and homogeneous cGi500 expression, we used targeted mutagenesis to integrate a Credependent cGi500 expression cassette into the R26 gene locus (Figure 1C middle). This gene locus is known for transcriptional accessibility in most if not all cell types throughout pre- and postnatal development (Soriano, 1999). Although technically more challenging, generation of R26 knock-in mice became a popular approach to express fluorescent proteins including biosensors (Zariwala et al., 2012; Abe et al., 2013; Batti et al., 2013). To increase biosensor expression above the relatively low levels that are usually achieved with the endogenous R26 


\section{(1) create binary mask}

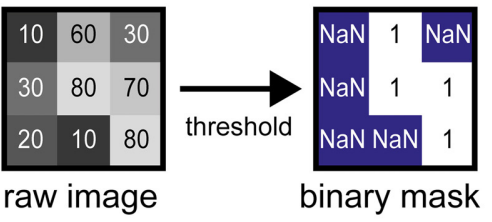

\section{(2) use binary mask (image segmentation)}

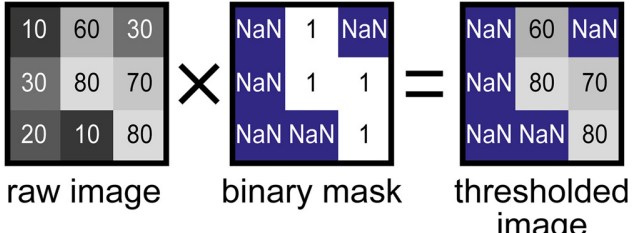

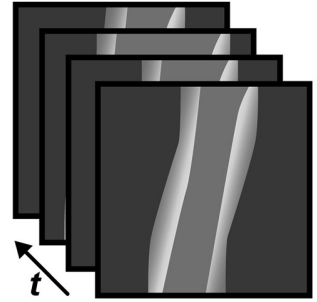

raw CFP time series

(1)

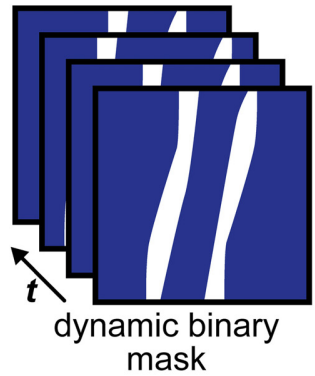

mask

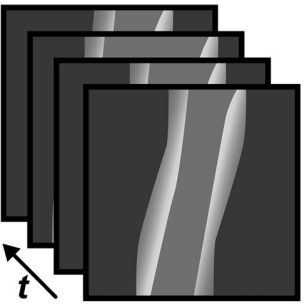

raw YFP time series

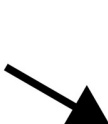

(2)

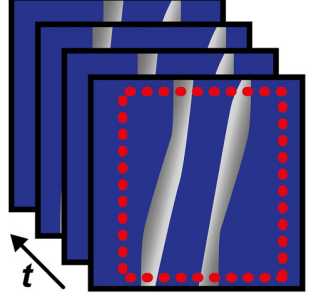

thresholded CFP time series

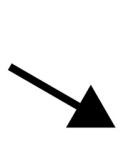

(2)

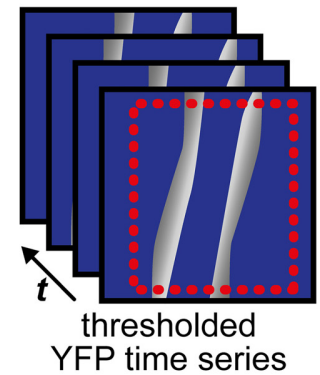

(3)

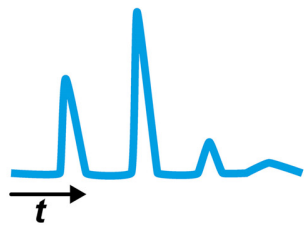

CFP intensities in a ROI over time

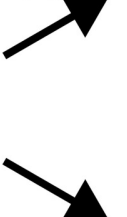

(4)

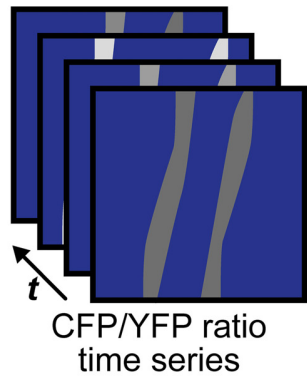

(3)

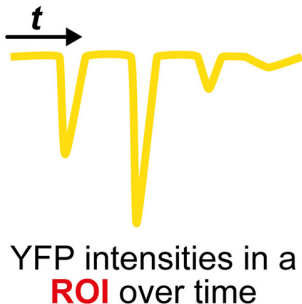

FIGURE 4 | Image segmentation approach to evaluate time-lapse series of moving structures. (1) A threshold is applied to the raw CFP time series to generate a dynamic binary mask. The raw YFP series can also be used, but only one mask (from CFP or from YFP) is required. Numbers within the raw images represent virtual pixel intensities; in this example, the threshold was set to an intensity of 50 . Now, target structures with intensities above the threshold receive the value "1" (white), while $\mathrm{dim} /$ non-fluorescent structures below the threshold receive the value "NaN" for "not a number" (blue), that does not equal "0." (2) Image segmentation is performed by multiplication of the same dynamic binary mask with the original raw CFP and YFP time series. Within segmented time series, pixels representing (bright) target structures retain their original intensities, while pixels representing dim/non-fluorescent structures are now set to non-numerical values ("NaN," blue). (3) CFP and YFP intensity changes are extracted from segmented time series in a region of interest (ROI, red) that contains the moving vessel wall throughout the whole time series. "NaN" pixels do not contribute to the ROl's mean intensities. (4) Segmented CFP and YFP time series are used to calculate pixel-by-pixel CFP/YFP ratio series. As background is set to "NaN," ratio series do not suffer from artifacts occurring when noisy images are divided by each other. The dynamic binary mask can also be used to estimate changes in vessel diameter (see Figure 3D) promoter in adult mice, the strong chicken actin/ $\beta$-globin (CAG) promoter (Niwa et al., 1991) can be integrated into the R26 knock-in transgene. Activation of sensor expression is achieved by breeding mice carrying the R26 knock-in transgene to cell type-specific Cre-transgenic mouse lines. This strategy should allow one to direct high and homogeneous levels of biosensor expression to almost any cell type of interest. We have generated R26 knock-in mice carrying the Cre-dependent 
R26-CAG-cGi500(L2) transgene (Thunemann et al., 2013b). We observed strong and tissue-specific cGi500 expression in mice generated by breeding R26-CAG-cGi500(L2) mice with tissue-specific Cre mice (Figure 2D, and Wen, Thunemann, Feil, unpublished results). This finding is in line with numerous reports on other Cre-inducible R26 knock-in transgenes (Casola, 2010). Furthermore, we transfected embryonic stem cells carrying the R26-CAG-cGi500(L2) transgene with a Cre expression plasmid to generate the permanently activated R26CAG-cGi500(L1) transgene (Figure 1C lower). Indeed, mice established from R26-CAG-cGi500(L1) embryonic stem cells showed strong cGi500 expression in virtually all tissues and cell types examined (Thunemann et al., 2013b). Importantly, use of the CAG promoter resulted in high cGi500 expression levels and sufficient fluorescence intensities for in vivo imaging experiments (Figures 2, 3, 5). We conclude that the wide use of the Cre/lox system and the availability of hundreds of Cre mouse lines combined with the positive experience using the R26 locus as knock-in target, the generation of biosensor-expressing mice via Cre/loxactivatable R26 knock-in transgenes is an attractive alternative to random transgenesis.

When working with biosensor-expressing cells, tissues, and mice, it is important to consider potential side effects of sensor expression. A biocompatible sensor should not interfere with normal physiology. The cGi500 sensor contains the cGMPbinding sites of cGK type I, but not its catalytic kinase domain nor its N-terminal region that interacts with various proteins (Figure 1B). Although we cannot formally exclude that cGi500 acts as an intracellular cGMP buffer, we did not observe any adverse effects of its expression on the health of cGi500-transgenic mice nor on the functions of cells isolated from these mice (Thunemann et al., 2013b). In this context, it is interesting to note that mice expressing a FRET-based $\mathrm{Ca}^{2+}$ sensor in a ubiquitous manner show only relatively mild phenotypes (Direnberger et al., 2012). However, when mice express a biosensor with enzymatic activity, for instance, myosin light chain kinase activity in MLCK $\mathrm{Ca}^{2+}$ indicator mice (Isotani et al., 2004), it might be important to exclude interference of the sensor with normal physiology by reducing its expression level as much as possible.

\section{IMAGING OF cGMP WITHIN THE CREMASTER MICROCIRCULATION}

The mouse cremaster is a relatively transparent skeletal muscle ( $\sim 200 \mu \mathrm{m}$ thick) that forms a pouch around the testis and contains resistance-type vessels. The first in vivo study of vascular responses within the cremaster was published in 1964 (Grant, 1964). Most current protocols for cremaster IVM, including those presented herein, follow the procedure described by Baez (1973), which includes the opening of the cremaster muscle, its detachment from the testicle, and the spreading of the cremaster to a flat sheet (Figure 2A). A number of studies used biosensors to visualize $\mathrm{Ca}^{2+}$ in endothelium and SMCs of cremaster arterioles. However, the functional role of cGMP in the cremaster microcirculation has so far been studied only indirectly, for instance, by analyzing vasodilation or vascular permeability in mouse lines with mutations of components of the cGMP signaling pathway (Koeppen et al., 2004; Chen et al., 2012).
Using the cGi500 sensor, we were able to visualize changes in cGMP concentrations in cremaster arterioles in vivo (Thunemann et al., 2013b). Experiments were performed with the R26-CAGcGi500(L1) mouse line showing ubiquitous cGi500 expression. Accordingly, we observed cGi500 expression in the vessel wall and in the striated muscle of the cremaster itself, in which vessels are embedded, as well as in circulating blood cells (Figures 2B,C,E, and data not shown). Application of the NO-releasing drugs sodium nitroprusside and DEA/NO, as well as adenosine and acetylcholine induced dilation of cremaster arterioles (Figure 2B, and data not shown). However, attempts to detect DEA/NOinduced cGMP signals in arterioles of the intact cremaster preparation (as shown in Figure 2B) failed due to strong cGi500 fluorescence in striated muscle (data not shown). After surgical removal of the skeletal muscle from arterioles (Figures 2C,E), robust $\mathrm{CFP} / \mathrm{YFP}$ ratio changes indicating reversible cGMP elevations could be observed in the vessel wall upon superfusion of DEA/NO, while adenosine did not cause a detectable cGMP elevation (Figure 2F). However, because vessels that were isolated from surrounding tissue apparently lost their basal tone and did not dilate upon drug superfusion, this experimental setup did not allow us to correlate cGMP levels with the extent of vasodilation. Moreover, because of ubiquitous cGi500 expression in R26-CAGcGi500(L1) mice, it is difficult to allocate cGMP signals to specific vascular cell types. Thus, this model has two limitations related to ubiquitous sensor expression. Firstly, FRET recordings are disturbed by fluorescence from striated muscle and, secondly, it is difficult to pinpoint cGMP signals to specific vascular cell types. Both problems could be avoided by cell type-specific cGi500 expression within distinct cell types of the cremaster microvasculature. Therefore, the Cre-responsive R26-CAG-cGi500(L2) mouse line is currently bred with endothelium- or SMC-specific Cre lines (Figure 2D, and data not shown). Furthermore, by using other Cre-transgenic lines, it should be possible to visualize cGMP signals in various other cell types, for instance, in leukocytes during inflammatory processes or in platelets during adhesion to the vessel wall and thrombus formation.

Advantages of the open cremaster model for IVM are its outstanding optical quality and relatively minor motion artifacts. The good accessibility of arterioles within the exteriorized cremaster allows direct drug delivery via superfusion or locally via glass pipettes. Applying drugs only to the tissue of interest and not systemically through intravenous infusion can be an advantage. Continuous superfusion almost instantaneously removes drugs, allowing for studies with many successive drug applications, for example, to generate concentration-response curves or to compare effects of different drugs. On the other hand, cremaster IVM requires supported breathing, continuous delivery of anesthetics, temperature maintenance with external heat sources, and constant superfusion with gassed salt solution. The time required to prepare an animal for imaging is around $1 \mathrm{~h}$, and the time for experiments is limited to about $6 \mathrm{~h}$ per animal.

\section{MULTIPHOTON cGMP IMAGING OF SUBCUTANEOUS VESSELS USING THE DORSAL SKINFOLD CHAMBER}

The DSC model (Figure 3A) has been established to study tumors implanted below the chamber window. In addition, this method 


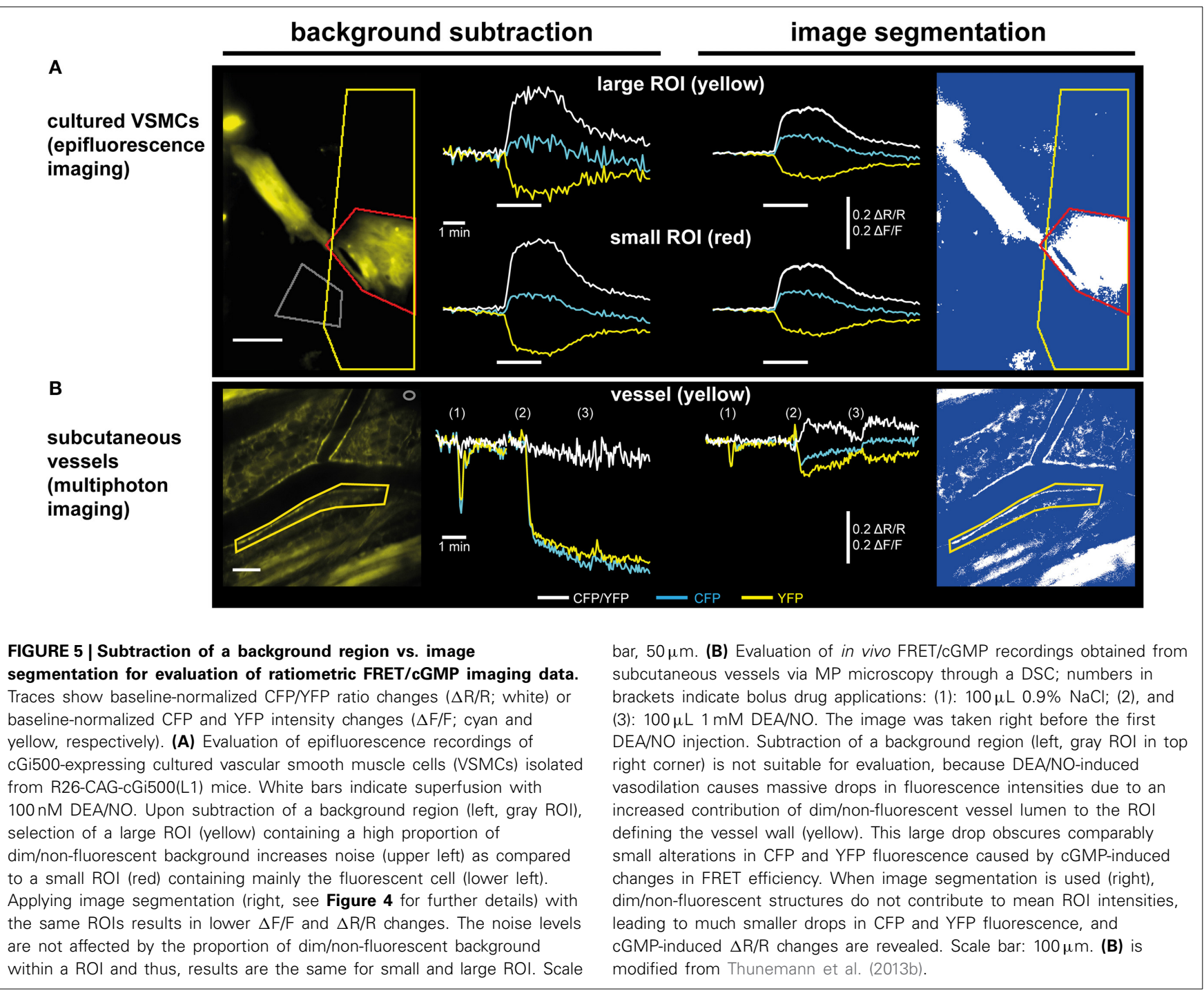

allows imaging of the tumor environment, for instance, to monitor changes in vascularization during tumor growth (Brown et al., 2001). IVM studies with DSCs were initially performed with bright field, epifluorescence, or confocal microscopy, but nowadays, MP microscopy is primarily used. MP excitation has several advantages over single photon excitation: out-of-focus fluorescence above or below the focal plane is avoided, excitation with pulsed infrared lasers allows for larger penetration depths (up to $\sim 0.5 \mathrm{~mm}$ tissue), and the infrared light causes comparatively mild damage to fluorescent molecules and surrounding tissue (Helmchen and Denk, 2005).

We implanted DSCs into R26-CAG-cGi500(L1) mice and used them for MP-FRET/cGMP imaging (Thunemann et al., 2013b). Subcutaneous vessels were readily visible and, in contrast to the cremaster model, we were able to identify regions where surrounding tissue did not interfere with the detection of FRET/cGMP signals from vessel walls (Figures 3B,E). Mice received vasoactive drugs as bolus injections via a tail vein catheter. In our experiments, 3-5 drug applications were performed over $15-30 \mathrm{~min}$. In studies with cultured cells

and cremaster arterioles, the initial steps in image evaluation are ROI definition and subtraction of a $\mathrm{dim} /$ non-fluorescent background region (see Figure 2E). When we subtracted intensities from a background region, vasodilation strongly confounded the analysis of CFP/YFP ratio signals within the vessel wall (Figure 5B left). Vasodilation led to an increase in the proportion of $\mathrm{dim} /$ non-fluorescent vascular lumen within the ROI that resulted in strong and parallel reductions of CFP and YFP fluorescence. These drops were much stronger than any intensity changes caused by cGMP binding to cGi500 (Figure 5B left). To overcome this problem, we performed image segmentation with a dynamic binary mask (Figure 4). Here, instead of subtracting a background region, structures with a brightness below a certain threshold are set to non-numeric values ("NaN"). These structures actually can be fluorescent; however, their brightness is lower compared to the target structure, i.e., the vessel wall. To compare subtraction of a background region and image segmentation, we applied both methods to cGi500 recordings in cultured cells. Subtraction of a background region results in greater changes of the baseline-normalized CFP/YFP ratio $(\Delta R / R)$ as 
compared to ratio changes obtained through image segmentation (Figure 5A, compare left and right traces), most likely as a direct result of different background treatment. However, when the dynamic binary mask is used, varying proportions of dim/nonfluorescent background within the ROI do not affect noise levels (Figure 5A, compare right traces from small and large ROI), which can be an advantage over the background subtraction method (Figure 5A, compare left traces from small and large ROI). Consequently, ROIs can now be defined to contain large and/or variable proportions of $\mathrm{dim} /$ non-fluorescent background without affecting signal quantification. Using this approach with DSC recordings markedly reduced the confounding drop of CFP and YFP fluorescence upon vasodilation that was observed when a background region was subtracted (Figure 5B). This alternative evaluation strategy eventually allowed us to detect cGMP transients in the vascular wall during vasodilation in vivo. Control injection of saline caused a transient vasodilation, most likely due to an increase in blood volume (data not shown) that was not associated with CFP/YFP ratio changes [Figure 5B, right, application (1)]. Injection of DEA/NO caused vasodilation (Figure 3E) that was associated with a clear $\Delta R / R$ increase indicating an increase of cGMP levels [Figures $3 \mathbf{E}$ and $5 \mathbf{B}$, right, applications (2) and (3)]. Relative vessel diameters were extracted from dynamic binary masks via kymographs (Figure 3D). The extent of NO-induced cGMP elevations measured with the cGi500 sensor correlated well with the extent of vasodilation (Figure 3E). Thus, data evaluation with the image segmentation method made it possible to observe cGMP transients in dilating vessels (Figures 3B-E).

The highest CFP/YFP ratio changes observed in the DSC model were relatively moderate $(\sim 10 \%)$ compared to changes recorded in the cremaster microvasculature ( $\sim 50 \%)$. Although we cannot exclude biological reasons, this discrepancy is likely caused by different ways of sensor excitation (single photon vs. MP) and data evaluation (treatment of background fluorescence). While image segmentation of DSC recordings was performed without background subtraction, non-specific fluorescence from a background region was subtracted in cremaster recordings. As Figure 5 clearly shows, the evaluation strategy strongly affects $\Delta R / R$ values. Therefore, quantitative comparisons are only feasible between experiments evaluated with the same strategy. Note that omitting background subtraction during image segmentation may introduce variability if the background varies considerably between experiments. We are working on further refinements of our evaluation approach and expect substantial improvements with the use of mice expressing cGi500 in a cell type-specific manner (see Figure 2D). Another difference was that single-photon excitation was used for cremaster imaging and MP excitation in the DSC model. For the latter, we tested excitation wavelengths between 820 and $880 \mathrm{~nm}$ and we have chosen $850 \mathrm{~nm}$ for best imaging results (data not shown). Due to overlapping excitation spectra of CFP and YFP and the non-linearity of MP excitation, MP lasers likely cause direct excitation of the acceptor fluorophore, YFP (Zipfel et al., 2003; Svoboda and Yasuda, 2006). We assume that direct YFP excitation as well as bleed-through of CFP emission into the YFP channel were, at least in part, responsible for the relatively low $\Delta R / R$ values observed during MP imaging in the DSC model. In order to apply correction algorithms for tissueintrinsic background fluorescence or spectral bleed-through, or to perform spectral unmixing, samples containing either no, or only the donor or the acceptor fluorophore need to be recorded (Van Rheenen et al., 2004; Thaler et al., 2005; Thaler and Vogel, 2006; Zhang et al., 2010; Zeug et al., 2012; Mauban et al., 2014). In addition, alternative methods to estimate FRET efficiencies such as fluorescence lifetime imaging microscopy (FLIM) are available (Yasuda et al., 2006; Levitt et al., 2009; Fruhwirth et al., 2011). However, recording appropriate donor- or acceptor-containing specimens, or using FLIM techniques with non-optimized FRET pairs can be challenging under in vivo conditions (Niesner and Hauser, 2011; Geiger et al., 2012; Kamioka et al., 2012).

In the DSC model, animals can be analyzed repeatedly for up to 5 weeks, which is an advantage over the cremaster model. The DSC model allows longitudinal studies in individual animals, for example, to follow disease progression. Thereby, animal numbers can be reduced, while statistical power is maintained. Another advantage over the cremaster model is that tissues within the DSC are not acutely touched when imaging is performed. Under the conditions described here, test drugs are delivered intravenously. Due to volume restrictions, this limits the number of drug applications during an imaging session to about three to five. Depending on the experimental question, systemic drug delivery might complicate data interpretation. If so, the DSC model can also be used for direct topical application of test compounds. To this end, the coverslip is carefully removed to visualize vessels and other structures while applying solutions that contain test compounds (Fukumura et al., 1997). A number of protocols exist to implant windows at different sites of the body (Jain et al., 2010; Ritsma et al., 2012). This flexibility allows studying cGMP and other signaling molecules in diverse vascular beds, such as cerebral vessels and the vasculature of abdominal or thoracic organs. For these experiments, the setup has to reach sufficient scanning speeds to resolve the kinetics of the biochemical/physiological processes under investigation. Acquisition speed is especially important when moving cells (e.g., in the blood) or structures (e.g., the beating heart) are analyzed. Here, camera-based systems have an advantage as they typically allow faster acquisition rates (Stephens and Allan, 2003). For some IVM applications, spinning disk systems might be a suitable alternative to MP systems (Jenne et al., 2011), particularly when combined with MP excitation, thereby achieving large penetration depths and fast acquisition rates (Shimozawa et al., 2013). Alternatively, resonant galvanometer mirror scanning enables MP microscopy at high speeds to monitor fast biological processes (Kirkpatrick et al., 2012).

\section{CONCLUSION AND OUTLOOK}

Correlative IVM of biochemical processes and physiological responses is a powerful technique to study cardiovascular biology in vivo. Although technically challenging, experiments performed in living animals are essential to analyze the functional relevance of proteins and signaling molecules in health and disease. We have generated and characterized transgenic mice expressing the FRET-based cGMP biosensor cGi500 in the vasculature. Indeed, intravital FRET imaging combined with an image-processing routine that accounts for tissue motion allowed 
us to monitor cGMP signals and associated vasodilation simultaneously in living mice. These and similar mouse models should be useful to investigate the crosstalk between vascular cGMP, cAMP, and $\mathrm{Ca}^{2+}$ signaling. For these and other studies, it will be important to proof the selectivity of the cGi500 sensor for detection of cGMP over cAMP in vivo, for example, by using cAMP-increasing agents and pharmacological/genetic inhibition of NO-induced cGMP synthesis. In the future, cGi500-expressing mice can be used to visualize cGMP selectively in defined cell types of the vessel wall, such as endothelial cells or SMCs, or to analyze cGMP signaling in mouse models of cardiovascular disorders. Another step to take is the precise quantification of cGMP concentrations within the vasculature under basal and stimulated conditions in vivo, as it has recently been described for $\mathrm{Ca}^{2+}$ (Mauban et al., 2014). The combination of genetic mouse models, optical biosensors, and state-of-the-art IVM methods should soon allow us to "watch" biochemistry, (patho-)physiology, and pharmacotherapy in the context of a living mammalian organism.

\section{ACKNOWLEDGMENTS}

We thank Barbara Birk and Rita Meurer for excellent technical assistance, Julia Kahn for help with DSC implantation, and Lai Wen for providing R26-CAG-cGi500(L2) mice. We want to acknowledge Ralf Jansen for providing imaging equipment, as well as FEI Munich and Visitron for support during establishment of cremaster IVM. The authors would like to thank Lai Wen and Markus Milde for providing unpublished data and reading the manuscript, as well as all members of the Feil laboratory for critical discussion. This work was supported by Open Access Publishing Fund of Tübingen University and the Deutsche Forschungsgemeinschaft (Robert Feil), the US Department of Defense Breast Cancer Research Innovator Award W81XWH10-1-0016 (Rakesh K. Jain) and US National Cancer Institute grants P01-CA080124 (Rakesh K. Jain and Dai Fukumura) and R01-CA096915 (Dai Fukumura).

\section{REFERENCES}

Abe, T., Sakaue-Sawano, A., Kiyonari, H., Shioi, G., Inoue, K., Horiuchi, T., et al. (2013). Visualization of cell cycle in mouse embryos with Fucci2 reporter directed by Rosa26 promoter. Development 140, 237-246. doi: 10.1242/dev.084111

Baez, S. (1973). An open cremaster muscle preparation for the study of blood vessels by in vivo microscopy. Microvasc. Res. 5, 384-394. doi: 10.1016/00262862(73)90054-X

Batti, L., Mukhtarov, M., Audero, E., Ivanov, A., Paolicelli, R. C., Zurborg, S., et al. (2013). Transgenic mouse lines for non-invasive ratiometric monitoring of intracellular chloride. Front. Mol. Neurosci. 6:11. doi: 10.3389/fnmol.2013.00011

Beavo, J. A., and Brunton, L. L. (2002). Cyclic nucleotide research—still expanding after half a century. Nat. Rev. Mol. Cell Biol. 3, 710-718. doi: 10.1038/nrm911

Berridge, M. J. (2008). Smooth muscle cell calcium activation mechanisms. J. Physiol. 586, 5047-5061. doi: 10.1113/jphysiol.2008.160440

Bhargava, Y., Hampden-Smith, K., Chachlaki, K., Wood, K. C., Vernon, J., Allerston, C. K., et al. (2013). Improved genetically-encoded, FlincG-type fluorescent biosensors for neural cGMP imaging. Front. Mol. Neurosci. 6:26. doi: 10.3389/fnmol.2013.00026

Brown, E. B., Campbell, R. B., Tsuzuki, Y., Xu, L., Carmeliet, P., Fukumura, D., et al. (2001). In vivo measurement of gene expression, angiogenesis and physiological function in tumors using multiphoton laser scanning microscopy. Nat. Med. 7, 864-868. doi: 10.1038/89997

Carvajal, J. A., Germain, A. M., Huidobro-Toro, J. P., and Weiner, C. P. (2000). Molecular mechanism of cGMP-mediated smooth muscle relaxation.
J. Cell. Physiol. 184, 409-420. doi: 10.1002/1097-4652(200009)184:3<409::AIDJCP16>3.0.CO;2-K

Casola, S. (2010). Mouse models for miRNA expression: the ROSA26 locus. Methods Mol. Biol. 667, 145-163. doi: 10.1007/978-1-60761-811-9_10

Chen, T. W., Wardill, T. J., Sun, Y., Pulver, S. R., Renninger, S. L., Baohan, A., et al. (2013). Ultrasensitive fluorescent proteins for imaging neuronal activity. Nature 499, 295-300. doi: 10.1038/nature 12354

Chen, W., Gassner, B., Borner, S., Nikolaev, V. O., Schlegel, N., Waschke, J., et al. (2012). Atrial natriuretic peptide enhances microvascular albumin permeability by the caveolae-mediated transcellular pathway. Cardiovasc. Res. 93, 141-151. doi: $10.1093 / \mathrm{cvr} / \mathrm{cvr} 279$

Clegg, R. M. (2009). "Förster resonance energy transfer-FRET what is it, why do it, and how it's done," in Laboratory Techniques in Biochemistry and Molecular Biology, ed T. W. J. Gadella (Amsterdam; Boston, MA: Elsevier), 1-57.

De Wit, C., Bolz, S. S., and Pohl, U. (2000). Interaction of endothelial autacoids in microvascular control. Z. Kardiol. 89 Suppl. 9:IX/113-116. doi: $10.1007 / \mathrm{s} 003920070015$

De Wit, C., Von Bismarck, P., and Pohl, U. (1994). Synergistic action of vasodilators that increase cGMP and cAMP in the hamster cremaster microcirculation. Cardiovasc. Res. 28, 1513-1518. doi: 10.1093/cvr/28.10.1513

Direnberger, S., Mues, M., Micale, V., Wotjak, C. T., Dietzel, S., Schubert, M., et al. (2012). Biocompatibility of a genetically encoded calcium indicator in a transgenic mouse model. Nat. Commun. 3:1031. doi: 10.1038/ncomms2035

Feil, R., Feil, S., and Hofmann, F. (2005). A heretical view on the role of NO and cGMP in vascular proliferative diseases. Trends Mol. Med. 11, 71-75. doi: 10.1016/j.molmed.2004.12.001

Feil, R., Lohmann, S. M., De Jonge, H., Walter, U., and Hofmann, F (2003). Cyclic GMP-dependent protein kinases and the cardiovascular system: insights from genetically modified mice. Circ. Res. 93, 907-916. doi: 10.1161/01.RES.0000100390.68771.CC

Fischmeister, R., Castro, L. R., Abi-Gerges, A., Rochais, F., Jurevicius, J., Leroy, J. et al. (2006). Compartmentation of cyclic nucleotide signaling in the heart: the role of cyclic nucleotide phosphodiesterases. Circ. Res. 99, 816-828. doi: 10.1161/01.RES.0000246118.98832.04

Francis, S. H., Blount, M. A., and Corbin, J. D. (2011). Mammalian cyclic nucleotide phosphodiesterases: molecular mechanisms and physiological functions. Physiol. Rev. 91, 651-690. doi: 10.1152/physrev.00030.2010

Fruhwirth, G. O., Fernandes, L. P., Weitsman, G., Patel, G., Kelleher, M., Lawler, K., et al. (2011). How förster resonance energy transfer imaging improves the understanding of protein interaction networks in cancer biology. Chemphyschem 12, 442-461. doi: 10.1002/cphc.2010 00866

Fukumura, D., Yuan, F., Endo, M., and Jain, R. K. (1997). Role of nitric oxide in tumor microcirculation. Blood flow, vascular permeability, and leukocyteendothelial interactions. Am. J. Pathol. 150, 713-725.

Geiger, A., Russo, L., Gensch, T., Thestrup, T., Becker, S., Hopfner, K. P., et al. (2012). Correlating calcium binding, Förster resonance energy transfer, and conformational change in the biosensor TN-XXL. Biophys. J. 102, 2401-2410. doi: 10.1016/j.bpj.2012.03.065

Grant, R. T. (1964). Direct observation of skeletal muscle blood vessels (rat cremaster). J. Physiol. 172, 123-137.

Helmchen, F., and Denk, W. (2005). Deep tissue two-photon microscopy. Nat. Methods 2, 932-940. doi: 10.1038/nmeth818

Hofmann, F., Feil, R., Kleppisch, T., and Schlossmann, J. (2006). Function of cGMPdependent protein kinases as revealed by gene deletion. Physiol. Rev. 86, 1-23. doi: 10.1152/physrev.00015.2005

Isotani, E., Zhi, G., Lau, K. S., Huang, J., Mizuno, Y., Persechini, A., et al. (2004). Real-time evaluation of myosin light chain kinase activation in smooth muscle tissues from a transgenic calmodulin-biosensor mouse. Proc. Natl. Acad. Sci. U.S.A. 101, 6279-6284. doi: 10.1073/pnas.0308742101

Jain, R. K., Munn, L. L., and Fukumura, D. (2002). Dissecting tumour pathophysiology using intravital microscopy. Nat. Rev. Cancer 2, 266-276. doi: $10.1038 / \mathrm{nrc778}$

Jain, R. K., Munn, L. L., and Fukumura, D. (2010). "Intravital microscopy of normal and diseased tissues in mice," in Live Cell Imaging: A Laboratory Manual, eds R. Goldman and D. Spector (Cold Spring Harbor, NY: Cold Spring Harbor Laboratory Press), 475-522.

Jenne, C. N., Wong, C. H., Petri, B., and Kubes, P. (2011). The use of spinning-disk confocal microscopy for the intravital analysis of platelet dynamics in response 
to systemic and local inflammation. PLoS ONE 6:e25109. doi: 10.1371/journal.pone.0025109

Kaestner, L., Scholz, A., Tian, Q., Ruppenthal, S., Tabellion, W., Wiesen, K., et al. (2014). Genetically encoded $\mathrm{Ca}^{2+}$ indicators in cardiac myocytes. Circ. Res. 114, 1623-1639. doi: 10.1161/CIRCRESAHA.114.303475

Kamioka, Y., Sumiyama, K., Mizuno, R., Sakai, Y., Hirata, E., Kiyokawa, E., et al. (2012). Live imaging of protein kinase activities in transgenic mice expressing FRET biosensors. Cell Struct. Funct. 37, 65-73. doi: 10.1247/csf.11045

Kardash, E., Bandemer, J., and Raz, E. (2011). Imaging protein activity in live embryos using fluorescence resonance energy transfer biosensors. Nat. Protoc. 6, 1835-1846. doi: 10.1038/nprot.2011.395

Kemp-Harper, B., and Feil, R. (2008). Meeting report: cGMP matters. Sci. Signal 1:pe12. doi: 10.1126/stke.19pe12

Kirkpatrick, N. D., Chung, E., Cook, D. C., Han, X., Gruionu, G., Liao, S., et al. (2012). Video-rate resonant scanning multiphoton microscopy: an emerging technique for intravital imaging of the tumor microenvironment. Intravital 1, 60-68. doi: 10.4161/intv.21557

Koeppen, M., Feil, R., Siegl, D., Feil, S., Hofmann, F., Pohl, U., et al. (2004). cGMP-dependent protein kinase mediates NO- but not acetylcholineinduced dilations in resistance vessels in vivo. Hypertension 44, 952-955. doi: 10.1161/01.HYP.0000147661.80059.ca

Kuhn, M. (2012). Endothelial actions of atrial and B-type natriuretic peptides. Br. J. Pharmacol. 166, 522-531. doi: 10.1111/j.1476-5381.2012.01827.x

Lee, S., Vinegoni, C., Sebas, M., and Weissleder, R. (2014). Automated motion artifact removal for intravital microscopy, without a priori information. Sci. Rep. 4:4507. doi: 10.1038/srep04507

Leunig, M., Yuan, F., Menger, M. D., Boucher, Y., Goetz, A. E., Messmer, K., et al. (1992). Angiogenesis, microvascular architecture, microhemodynamics, and interstitial fluid pressure during early growth of human adenocarcinoma LS174T in SCID mice. Cancer Res. 52, 6553-6560.

Levitt, J. A., Matthews, D. R., Ameer-Beg, S. M., and Suhling, K. (2009). Fluorescence lifetime and polarization-resolved imaging in cell biology. Curr. Opin. Biotechnol. 20, 28-36. doi: 10.1016/j.copbio.2009.01.004

Li, L., Miano, J. M., Mercer, B., and Olson, E. N. (1996). Expression of the SM22alpha promoter in transgenic mice provides evidence for distinct transcriptional regulatory programs in vascular and visceral smooth muscle cells. J. Cell Biol. 132, 849-859. doi: 10.1083/jcb.132.5.849

Lincoln, T. M., Dey, N., and Sellak, H. (2001). Invited review: cGMP-dependent protein kinase signaling mechanisms in smooth muscle: from the regulation of tone to gene expression. J. Appl. Physiol. 91, 1421-1430.

Mauban, J. R., Fairfax, S. T., Rizzo, M. A., Zhang, J., and Wier, W. G. (2014). A method for noninvasive longitudinal measurements of $\left[\mathrm{Ca}^{2+}\right]$ in arterioles of hypertensive optical biosensor mice. Am. J. Physiol. Heart Circ. Physiol. 307, H173-H181. doi: 10.1152/ajpheart.00182.2014

Mauban, J. R., Zacharia, J., Zhang, J., and Wier, W. G. (2013). Vascular tone and $\mathrm{Ca}(2+)$ signaling in murine cremaster muscle arterioles in vivo. Microcirculation 20, 269-277. doi: 10.1111/micc. 12025

Morgado, M., Cairrao, E., Santos-Silva, A. J., and Verde, I. (2012). Cyclic nucleotide-dependent relaxation pathways in vascular smooth muscle. Cell Mol. Life Sci. 69, 247-266. doi: 10.1007/s00018-011-0815-2

Muzumdar, M. D., Tasic, B., Miyamichi, K., Li, L., and Luo, L. (2007). A global double-fluorescent Cre reporter mouse. Genesis 45, 593-605. doi: 10.1002/dvg.20335

Nausch, L. W., Ledoux, J., Bonev, A. D., Nelson, M. T., and Dostmann, W. R. (2008), Differential patterning of cGMP in vascular smooth muscle cells revealed by single GFP-linked biosensors. Proc. Natl. Acad. Sci. U.S.A. 105, 365-370. doi: 10.1073/pnas.0710387105

Newman, R. H., Fosbrink, M. D., and Zhang, J. (2011). Genetically encodable fluorescent biosensors for tracking signaling dynamics in living cells. Chem. Rev. 111, 3614-3666. doi: 10.1021/cr100002u

Niesner, R. A., and Hauser, A. E. (2011). Recent advances in dynamic intravital multi-photon microscopy. Cytometry A 79, 789-798. doi: 10.1002/cyto.a.21140

Nikolaev, V. O., and Lohse, M. J. (2009). Novel techniques for real-time monitoring of cGMP in living cells. Handb. Exp. Pharmacol. 191, 229-243. doi: 10.1007/9783-540-68964-5_11

Nikolaev, V. O., Moshkov, A., Lyon, A. R., Miragoli, M., Novak, P., Paur, H., et al. (2010). Beta2-adrenergic receptor redistribution in heart failure changes cAMP compartmentation. Science 327, 1653-1657. doi: 10.1126/science.11 85988
Niwa, H., Yamamura, K., and Miyazaki, J. (1991). Efficient selection for highexpression transfectants with a novel eukaryotic vector. Gene 108, 193-199. doi: 10.1016/0378-1119(91)90434-D

Pitkeathly, W. T., Poulter, N. S., Claridge, E., and Rappoport, J. Z. (2012). Autoalign-multi-modality fluorescence microscopy image co-registration. Traffic 13, 204-217. doi: 10.1111/j.1600-0854.2011.01309.x

Ritsma, L., Ponsioen, B., and Rheenen, J. (2012). Intravital imaging of cell signaling in mice. Intravital 1, 2-10. doi: 10.4161/intv.20802

Russwurm, M., Mullershausen, F., Friebe, A., Jager, R., Russwurm, C., and Koesling, D. (2007). Design of fluorescence resonance energy transfer (FRET)based cGMP indicators: a systematic approach. Biochem. J. 407, 69-77. doi: 10.1042/BJ20070348

Schlossmann, J., and Schinner, E. (2012). cGMP becomes a drug target. Naunyn Schmiedebergs Arch. Pharmacol. 385, 243-252. doi: 10.1007/s00210-0120730-6

Schneider, C. A., Rasband, W. S., and Eliceiri, K. W. (2012). NIH Image to ImageJ: 25 years of image analysis. Nat. Methods 9, 671-675. doi: 10.1038/ nmeth.2089

Shimozawa, T., Yamagata, K., Kondo, T., Hayashi, S., Shitamukai, A., Konno, D., et al. (2013). Improving spinning disk confocal microscopy by preventing pinhole cross-talk for intravital imaging. Proc. Natl. Acad. Sci. U.S.A. 110, 3399-3404. doi: 10.1073/pnas.1216696110

Somlyo, A. P., and Somlyo, A. V. (2000). Signal transduction by G-proteins, rho-kinase and protein phosphatase to smooth muscle and non-muscle myosin II. J. Physiol. 522 Pt 2, 177-185. doi: 10.1111/j.1469-7793.2000.t01-200177.x

Soriano, P. (1999). Generalized lacZ expression with the ROSA26 Cre reporter strain. Nat. Genet. 21, 70-71. doi: 10.1038/5007

Stangherlin, A., and Zaccolo, M. (2012). cGMP-cAMP interplay in cardiac myocytes: a local affair with far-reaching consequences for heart function. Biochem. Soc. Trans. 40, 11-14. doi: 10.1042/BST20110655

Stephens, D. J., and Allan, V. J. (2003). Light microscopy techniques for live cell imaging. Science 300, 82-86. doi: 10.1126/science.1082160

Svoboda, K., and Yasuda, R. (2006). Principles of two-photon excitation microscopy and its applications to neuroscience. Neuron 50, 823-839. doi: 10.1016/j.neuron.2006.05.019

Tallini, Y. N., Brekke, J. F., Shui, B., Doran, R., Hwang, S. M., Nakai, J., et al. (2007). Propagated endothelial $\mathrm{Ca}^{2+}$ waves and arteriolar dilation in vivo: measurements in Cx40BAC GCaMP2 transgenic mice. Circ. Res. 101, 1300-1309. doi: 10.1161/CIRCRESAHA.107.149484

Thaler, C., Koushik, S. V., Blank, P. S., and Vogel, S. S. (2005). Quantitative multiphoton spectral imaging and its use for measuring resonance energy transfer. Biophys. J. 89, 2736-2749. doi: 10.1529/biophysj.105.061853

Thaler, C., and Vogel, S. S. (2006). Quantitative linear unmixing of CFP and YFP from spectral images acquired with two-photon excitation. Cytometry A 69, 904-911. doi: 10.1002/cyto.a.20267

Thunemann, M., Fomin, N., Krawutschke, C., Russwurm, M., and Feil, R. (2013a) Visualization of cGMP with cGi biosensors. Methods Mol. Biol. 1020, 89-120. doi: 10.1007/978-1-62703-459-3_6

Thunemann, M., Wen, L., Hillenbrand, M., Vachaviolos, A., Feil, S., Ott, T., et al. (2013b). Transgenic mice for cGMP imaging. Circ. Res. 113, 365-371. doi 10.1161/CIRCRESAHA.113.301063

Tian, L., Hires, S. A., Mao, T., Huber, D., Chiappe, M. E., Chalasani, S. H., et al. (2009). Imaging neural activity in worms, flies and mice with improved GCaMP calcium indicators. Nat. Methods 6, 875-881. doi: 10.1038/ nmeth. 1398

Tsai, E. J., and Kass, D. A. (2009). Cyclic GMP signaling in cardiovascular pathophysiology and therapeutics. Pharmacol. Ther. 122, 216-238. doi: 10.1016/j.pharmthera.2009.02.009

Van Rheenen, J., Langeslag, M., and Jalink, K. (2004). Correcting confocal acquisition to optimize imaging of fluorescence resonance energy transfer by sensitized emission. Biophys. J. 86, 2517-2529. doi: 10.1016/S0006-3495(04) 74307-6

Weigert, R., Porat-Shliom, N., and Amornphimoltham, P. (2013). Imaging cell biology in live animals: ready for prime time. J. Cell Biol. 201, 969-979. doi: $10.1083 /$ jcb. 201212130

Wier, W. G. (2014). More in vivo experimentation is needed in cardiovascular physiology. Am. J. Physiol. Heart Circ. Physiol. 307, H121-H123. doi: 10.1152/ajpheart.00326.2014 
Yasuda, R., Harvey, C. D., Zhong, H., Sobczyk, A., Van Aelst, L., and Svoboda, K. (2006). Supersensitive Ras activation in dendrites and spines revealed by two-photon fluorescence lifetime imaging. Nat. Neurosci. 9, 283-291. doi: 10.1038/nn1635

Zaccolo, M., Cesetti, T., Di Benedetto, G., Mongillo, M., Lissandron, V., Terrin, A., et al. (2005). Imaging the cAMP-dependent signal transduction pathway. Biochem. Soc. Trans. 33, 1323-1326. doi: 10.1042/BST20051323

Zaccolo, M., and Movsesian, M. A. (2007). cAMP and cGMP signaling cross-talk: role of phosphodiesterases and implications for cardiac pathophysiology. Circ. Res. 100, 1569-1578. doi: 10.1161/CIRCRESAHA.106.144501

Zariwala, H. A., Borghuis, B. G., Hoogland, T. M., Madisen, L., Tian, L., De Zeeuw, C. I., et al. (2012). A Cre-dependent GCaMP3 reporter mouse for neuronal imaging in vivo. J. Neurosci. 32, 3131-3141. doi: 10.1523/JNEUROSCI.446911.2012

Zeug, A., Woehler, A., Neher, E., and Ponimaskin, E. G. (2012). Quantitative intensity-based FRET approaches-a comparative snapshot. Biophys. J. 103, 1821-1827. doi: 10.1016/j.bpj.2012.09.031

Zhang, J., Chen, L., Raina, H., Blaustein, M. P., and Wier, W. G. (2010). In vivo assessment of artery smooth muscle $\left[\mathrm{Ca}^{2+}\right] \mathrm{i}$ and MLCK activation in FRET-based biosensor mice. Am. J. Physiol. Heart Circ. Physiol. 299, H946-H956. doi: 10.1152/ajpheart.00359.2010
Zipfel, W. R., Williams, R. M., and Webb, W. W. (2003). Nonlinear magic: multiphoton microscopy in the biosciences. Nat. Biotechnol. 21, 1369-1377. doi: $10.1038 /$ nbt899

Conflict of Interest Statement: The authors declare that the research was conducted in the absence of any commercial or financial relationships that could be construed as a potential conflict of interest.

Received: 16 July 2014; accepted: 23 September 2014; published online: 14 October 2014.

Citation: Thunemann M, Schmidt K, de Wit C, Han X, Jain RK, Fukumura D and Feil $R$ (2014) Correlative intravital imaging of cGMP signals and vasodilation in mice. Front. Physiol. 5:394. doi: 10.3389/fphys.2014.00394

This article was submitted to Cardiac Electrophysiology, a section of the journal Frontiers in Physiology.

Copyright (c) 2014 Thunemann, Schmidt, de Wit, Han, Jain, Fukumura and Feil. This is an open-access article distributed under the terms of the Creative Commons Attribution License (CC BY). The use, distribution or reproduction in other forums is permitted, provided the original author(s) or licensor are credited and that the original publication in this journal is cited, in accordance with accepted academic practice. No use, distribution or reproduction is permitted which does not comply with these terms. 